\title{
Analytical Evaluation of the Clinical Chemistry Analyzer AU 480 Beckman Coulter
}

\author{
Nardos Abebe \\ Bethzatha Advanced Medical Laboratory, Quality Assurance Department, Addis Ababa, Ethiopia \\ Email address: \\ nardosabebe799@gmail.com

\section{To cite this article:} \\ Nardos Abebe. Analytical Evaluation of the Clinical Chemistry Analyzer AU 480 Beckman Coulter. European Journal of Clinical and \\ Biomedical Sciences. Vol. 2, No. 6, 2016, pp. 59-62. doi: 10.11648/j.ejcbs.20160206.11
}

Received: October 12, 2016; Accepted: November 8, 2016; Published: December 10, 2016

\begin{abstract}
Background: Method verification is a one-time process to determine performance characteristics before a test system is utilized for patient testing. Objective: To evaluate the analytical performance of five analytes-glucose (glu), cholesterol (chol), creatinine (crea), aspartate aminotransferase, (AST) and alanine aminotransferase (ALT) on AU 480 Beckman Coulter clinical chemistry analyzer. Methods and Materials: Analytical evaluation of analyzer included the determination of within-run, within-laboratory imprecision, and Trueness. Beckman Coulter control level-one and control level-two, near medical decision points were used. It was done according to (CLSI) clinical laboratory standard institute (EP15-A2), which suggests two levels, run 3 times per run for 5 days (15replicates in all). Result: The results showed low within-run imprecision (level 1-ALT $=1.5 \%, \mathrm{AST}=1.3 \%$, chol $=0.5 \%$, crea $=0.2 \%$, glu=0.7\%, level $2-\mathrm{ALT}=1.0 \%, \mathrm{AST}=1.3 \%$, chol $=0.6 \%$, crea $=0.9 \%$, glu $=0.4 \%$ ) and within-laboratory imprecision (level 1 -ALT $=2.6 \%$, AST $=0.9 \%$, chol $=0.7 \%$, crea $=0.2 \%$, $\mathrm{glu}=0.8 \%$, level $2-\mathrm{ALT}=1.9 \%$, AST $=1.0 \%$, chol $=0.6 \%$, crea $=0.8 \%$, glu=0.5\%)and the assigned values were found to be within trueness verification intervals (level 1 at 95\% CI: ALT=46.5U/L[22.7-66.6], AST=50.4U/L[29.1-76.5], chol=156mg/dl[107.1197.1], crea $=1.19 \mathrm{mg} / \mathrm{dl}$ [0.96-2.03], glu=103mg/dl [69.8-136.3]) and (level 2 at 95\% CI: ALT=133U/L [60.5-187.3], $\mathrm{AST}=148 \mathrm{U} / \mathrm{L} \quad[81.3-220.3], \quad \mathrm{chol}=285 \mathrm{mg} / \mathrm{dl} \quad[186.2-349.8], \quad \mathrm{crea}=5.35 \mathrm{mg} / \mathrm{dl} \quad[3.46-8.3], \mathrm{glu}=239 \mathrm{mg} / \mathrm{dl} \quad[163-320.5])$. Conclusion: AU 480 Beckman Coulter clinical chemistry analyzer is suitable for analytical measurement of analytes-AST, ALT, cholesterol, creatinine, and glucose. If laboratories use CLSI and other guidelines, which are published on issues of biological variations, in addition to the manufacturer's claims, it may help laboratories not to waste unnecessary time and money for repeating of experiment.
\end{abstract}

Keywords: AU 480 Beckman Coulter, Trueness, Within-Run Imprecision, Within-Laboratory Imprecision

\section{Introduction}

Medical laboratories provide information and services that contribute to maximizing the effective delivery of care in today's complex healthcare system. Laboratory result enables physicians and other healthcare professionals to make appropriate evidence-based diagnostic or therapeutic decisions for their patients. In addition, laboratory results have a direct impact on many aspects of patient care including, but not limited to, length of stay, patient safety, resource utilization, and customer satisfaction [1].

In view of that, medical laboratories carrying out all types of measurements are increasingly being asked to evaluate their methods [2, 3, 4]. For medical laboratories, evaluating methods are now an accreditation requirement as specified by international standards such as ISO 15189:2012, and, In Ethiopia, ES-ISO 15189:2013 standards for medical laboratories $[5,6]$. As well, evaluation should be done before the introduction of the new analyzer into the routine use in order to confirm declared specifications of the analytical methods $[5,6]$. With this regard, the current study describes the analytical performance evaluation the equipment AU 480 Beckman Coulter Clinical Chemistry analyzer on five parameters.

\section{Methods and Materials}

\subsection{Clinical Chemistry Analyzer and Analytes}

AU480 (Beckman Coulter, Tokyo, Japan) is an ideal chemistry analyzer for small- to medium-sized hospitals and 
laboratories. It is fully automated analyzer where measurements are carried out using four different assay types of endpoint, rate, fixed point and indirect ion selective electrode, with analytical methods of colorimetry, turbidimetry, latex agglutination, and homogeneous enzyme immuno assay in serum, urine cerebrospinal fluid, and other body fluids samples. The analyzer can process random access throughput of up to 400 photometric tests per hour (up to 800 with electrolytes), with reduced sample volume and userfriendly operation.

The tested analytes were as follows: creatinine, glucose, cholesterol, aspartate aminotransferase (AST), alanine aminotransferase (ALT), and testing was conducted at clinical chemistry department of Bethzatha Advanced Medical Laboratory, Addis Ababa, Ethiopia.

\subsection{Analytical Evaluation}

Analytical evaluation of analyzer included the determination of within-run and within-laboratory imprecision, and Trueness. The evaluation was done according to clinical laboratory standard institute (CLSI) guidelines of EP15-A2. Within-run and within-laboratory imprecision were used to indicate the disagreement among a set of replicate measurements when all measurements are made under identical conditions (or within a single run of a procedure) and the disagreement among replicate measurements over a longer time respectively. Trueness is conformance to a true value, accepted standard, or expected value.

\subsection{Materials and Procedure for Experiments}

Since precision and trueness are directly related to concentration, Beckman Coulter control level-one and control level-two, near medical decision points were used. It was done according to CLSI protocol (EP15-A2), which suggests two levels, run 3 times per run for 5 days (15 replicates in all)

\subsection{Statistical Analysis and Procedure}

Statistical methods for imprecision included calculation of mean and coefficient of variation $(\% \mathrm{CV})$. Along with the mean and $\% \mathrm{CV}$, verification values (chi square) were calculated for imprecision's that were greater than the manufacturer's limit (shown below in equation 1 to equation 6). Besides, calculations of verification limit were included for the trueness (shown below in equation 7 to 12). The level of significance was set at $\mathrm{P}<0.05$. Finally, Statistical analysis was performed according to the mentioned below formulas using Microsoft Excel 2007.

Beckman Coulter control level one and Beckman Coulter level two were used. The procedure has been described in detailed elsewhere [7]. The within-run precision, withinlaboratory, and trueness for each analyte were as follows:

Within-run precision $\left(\mathrm{S}_{\mathrm{r}}\right)$

$$
S r=\sqrt{\frac{\sum_{d=1}^{D} \sum_{i=1}^{n}(X d i-X d)^{2}}{D(n-1)}}
$$

Where $\mathrm{D}=$ total number of days (five), $\mathrm{n}=$ total number of replicates per day (three), $x d i=$ result of $i$ replicate for day $d$ and $\overline{\bar{x}} \mathrm{~d}=$ average of all results for $\mathrm{d}$ days.

Within-Laboratory Precision $\left(\mathrm{S}_{1}\right)$

First calculated the variance term, $\mathrm{S}_{\mathrm{b}}{ }^{2}$, for the daily mean using.

$$
S b=\frac{\sum_{d=1}^{D}(\bar{x} d-\bar{x})^{2}}{D-1}
$$

Where $\bar{x} d$ average of all results for day $d\left(\bar{x}_{1}\right.$ is average for day 1 and $\overline{\bar{x}}=$ average of all results.

$$
S l=\sqrt{(n-1) / n * S r^{2}+S b^{2}}
$$

Where $n=3$

Following calculation of $\mathrm{Sr}$ and $\mathrm{Sl}$, statistical test was done for (when calculated $\mathrm{Sl} / \mathrm{Sr}>$ claimed $\sigma \mathrm{l} / \sigma \mathrm{r}$ ) as follows:

Calculated the degrees of freedom for within-run precision, $V$ for 5 days (D) and 3 replicates (n): $V=D(n-1)$, $\mathrm{V}=10$; Then determined the $(1-\alpha / \ell)$ percentage point, $\mathrm{C}$, of the Chi-Square distribution with 10 degrees of freedom. Here, $\alpha=5 \%$, and $\ell$ was 2 . $C=20.48$; finally calculated Chisquare as:

$$
\text { Chi }- \text { square }=\sigma r * \sqrt{C} / \sqrt{V}
$$

Calculated the effective degrees of freedom, $\mathrm{T}$, for the within-laboratory precision estimate, for 5 days duration and 3 replicates per run:

$$
T=\frac{((\mathrm{n}-1) * \operatorname{Sr} 2+(\mathrm{n} * \mathrm{Sb} 2)) 2}{(\mathrm{n}-1) / \mathrm{D} * \operatorname{Sr} 4+(\mathrm{n} 2 *(\mathrm{~S} 2 \mathrm{~b}) 2 / \mathrm{D}-1}
$$

Then determined the $(1-\alpha / \ell)$ percentage point, $C$, of the Chi-Square distribution with $\mathrm{T}$ degrees of freedom. Here, $\alpha$ was $5 \%$, and $\ell$ was 2 . Finally calculated verification values as:

$$
\text { Chi }- \text { square }=\sigma l * \sqrt{C} /_{\sqrt{T}}
$$

Trueness

$$
\begin{gathered}
\text { Xsum }=\sum_{\mathrm{i}=1}^{\mathrm{n} 10} \mathrm{xi}=(\mathrm{x} 1+\cdots+\mathrm{x} 10) \\
X a v=\sum_{\mathrm{i}=1}^{\mathrm{n} 10} \mathrm{xi}=(\mathrm{x} 1 . .+\mathrm{x} 10) / 10 \\
S D=\sqrt{\sum_{i=1}^{n 10}(x i-x \mathrm{av})^{2} / 9} \\
S D E=\frac{S D}{\sqrt{10}}
\end{gathered}
$$

$$
\mathrm{Sa}=\frac{C}{2} \text { Where } \mathrm{C}=\text { upper limit-Lower limit }
$$

Then, determined the $(100-\alpha / 2)$ percent point, $t$, of the $t-$ distribution with $2 n-1$ degrees of freedom. Here, $n=10$ 
represented the number of samples tested and 2 represented the number of replicates. $\alpha=5 \%$ and, the $(100-\alpha / 2)$ point of the t-distribution with 19 degrees of freedom was 2.093 .

$$
X a v \pm 2.093 * \sqrt{S D E^{2}+\mathrm{Sa}^{2}}
$$

\section{Results}

Performance analytical evaluation of five parameters, Creatinine, glucose, ALT, AST and cholesterol was carried out on equipment, AU 480 Beckman Coulter Clinical Chemistry analyzer. Standardized methodology and protocols were used on the mentioned equipment (AU 480) as per kit inserts or/and manufacturer's manual and procedural instructions. The coefficient of variation for within-run imprecision ranged from $0.2 \%-1.5 \%$ and from $0.4 \%-1.3 \%$ for level one and level two respectively as shown in table one. On top of the within-run imprecision, coefficient of variation for within-laboratory imprecision ranged from $0.2 \%-2.6 \%$ and from $0.5 \%-1.9 \%$ for level one and level two respectively as Shown in the same table. Finally, when it comes to the second type performance analytical evaluation, trueness, verification limit at $95 \%$ confidence interval were as follow: creatinine-0.96-2.03mg/dl, cholesterol-107.1-197.1mg/dl and ALT-22.7-66.6mg/dl as detailed below in table two.

Table 1. Manufacturer's specification of imprecision for the tested analytes, day-to-day and within-run imprecision.

\begin{tabular}{|c|c|c|c|c|c|c|c|c|}
\hline \multirow[t]{2}{*}{ Analyte } & \multicolumn{2}{|c|}{$\begin{array}{l}\text { Manufacturer's within run } \\
\text { Imprecision(CV\%) }\end{array}$} & \multicolumn{2}{|c|}{$\begin{array}{l}\text { Manufacturer's within laboratory } \\
\text { Imprecision (CV\%) }\end{array}$} & \multicolumn{2}{|c|}{$\begin{array}{l}\text { Verified Within run } \\
\text { imprecision CV\% }\end{array}$} & \multicolumn{2}{|c|}{$\begin{array}{l}\text { Verified Within lab } \\
\text { imprecision }(\mathrm{CV} \%)\end{array}$} \\
\hline & Level 1 & Level 2 & Level 1 & Level 2 & Level 1 & Level 2 & Level 1 & Level 2 \\
\hline ALT & 2.95 & 1.43 & 3.16 & 1.78 & 1.5 & 1.0 & 2.6 & 1.9 \\
\hline $\mathrm{AST}$ & 1.93 & 0.84 & 4.23 & 1.35 & 1.30 & 1.30 & 0.90 & 1.0 \\
\hline Cholesterol & 0.91 & 0.72 & 1.06 & 1.45 & 0.50 & 0.60 & 0.7 & 0.60 \\
\hline Creatinine & 0.96 & 0.74 & 2.48 & 1.54 & 0.20 & 0.9 & 0.20 & 0.80 \\
\hline Glucose & 0.70 & 0.54 & 1.25 & 0.97 & 0.70 & 0.40 & 0.80 & 0.50 \\
\hline
\end{tabular}

Table 2. Verification limit of verified reference material and the reference material target value.

\begin{tabular}{lllll}
\hline \multirow{2}{*}{ Analyte } & \multirow{2}{*}{ Method } & \multicolumn{2}{l}{ Reference Material Target } & \multicolumn{2}{l}{ Verification Limit of verified Reference Material } \\
\cline { 3 - 4 } & & Level 1 & Level 2 & Level 1 \\
\hline ALT & IFCC without Pyridoxal Phosphate & 46.5 & 133 & $22.7-66.6$ \\
AST & IFCC without Pyridoxal Phosphate & 50.4 & 148 & $29.1-76.5$ \\
Cholesterol & CHO-POD & 156 & 285 & $107.1-197.1$ \\
Creatinine & Jaffe IDMS traceable & 1.19 & 5.35 & $0.96-2.03$ \\
Glucose & HK G6P-DH & 103 & 239 & $69.8-187.3$ \\
\hline
\end{tabular}

\section{Discussion}

Amongst the calculated within- run imprecision's, all analytes, except, glucose for level 1, ASTand Creatinine for level 2 were less than the manufacturer's claimed CV. Hence, AU 480 chemistry analyzer demonstrated precision consistent with the manufacturer's claim, which is shown in table one. Whilst, the within-run imprecision, for the analytes, i.e., glucose, AST and creatinine were greater than the manufacturer's claimed coefficient of variation. Yet, it does not mean that, AU 480 chemistry analyzer did not demonstrated precision consistent with the manufacturer's claims or does not mean that verifications were failed.

On CLSI guideline of EP15-A2, it has been pointed up asthe user's within run imprecision can be larger than the manufacturer's claim and not be statistically different from the claim [4]. And, it has also been pointed up on the same guideline as- if the calculated within run imprecision $\mathrm{CV}$ is larger than the manufacturer's claim, test whether it is statistically significantly larger (really different) [4]. Accordingly, verification values were calculated for analytes, i.e., (glucose=1.2), $(\mathrm{AST}=1.4)$ and (creatinine $=0.93)$. Thus, as the result of high verification values (compared to calculated $\% \mathrm{CV}$ ), the claims were verified.

Of the within-laboratory imprecision, only ALT level two has greater coefficient of variation value when compared to the manufacturer's claim. Alike the within-run verification, verification value was calculated for ALT, which was 2.9. As a result, all claims were verified. Likewise, all the five analytes' imprecision were comparable to the desirable specification for imprecision and the recommended biological variations as shown in table three $[8,9,10]$.

Table 3. Desirable specification for imprecision derived from intra-, interindividual biologic variation.

\begin{tabular}{|c|c|c|c|}
\hline \multirow[t]{2}{*}{ Analyte } & \multicolumn{2}{|c|}{ Biological variation (CV\%) } & \multirow[b]{2}{*}{$\begin{array}{l}\text { Desirable specification } \\
\text { for imprecision }(\mathrm{CV} \%)\end{array}$} \\
\hline & $\begin{array}{l}\text { within- } \\
\text { subject }\end{array}$ & $\begin{array}{l}\text { between- } \\
\text { subjects }\end{array}$ & \\
\hline ALT & 19.4 & 41.6 & $9.7 \%$ \\
\hline AST & 12.3 & 23.1 & $6.15 \%$ \\
\hline Cholesterol & 5.95 & 15.3 & $2.98 \%$ \\
\hline Creatinine & 5.96 & 14.7 & $2.98 \%$ \\
\hline Glucose & 5.6 & 7.5 & $2.98 \%$ \\
\hline
\end{tabular}

Finally, the current study found, allthe calculated verification intervals included the assigned values. EP15-A2 guideline explicate that if the verification interval includes the assigned value, then the manufacturer's claim for trueness is verified. For that reason, the manufacturers' claimed for trueness's were verified for the AU 480 Beckman Coulter on the five parameters- Creatinine, Glucose, ALT, AST and Cholesterol. 


\section{Conclusion and Recommendation}

AU 480 clinical chemistry analyzer is suitable for analytical measurement of analytes-AST, ALT, cholesterol, creatinine, and glucose. In conclusion, if laboratories use CLSI and other guidelines, which is published on issues of biological variations, in addition to the manufacturer's claims, it may help laboratories not to waste unnecessary time and money for repeating of experiment.

\section{Competing Interests}

The author declares that there is no competing interest.

\section{Author's Contribution and Information}

Nardos Abebe: Design the project, perform the statistical analysis, and prepare the manuscript.

\section{Acknowledgements}

I greatly acknowledge Bethzatha Advanced Medical Laboratory, Addis Ababa, Ethiopia. I am also indebtedto my colleagues- Mr. Megerssa Hailu, Mr. Tibebe Adinew and Mr. Desalegn Adissie, who gave valuable comments on the manuscript.

\section{References}

[1] Forsman Rodney S. The Value of the Laboratory Professional in the Continuum of Care. Clinical leadership and management review, 370-373. Nov/Dec 2002.

[2] Juricek, J, L. Derek, A. Unic, T. Serdar, D. Marijancevic, M.
Zivkovic and Z. Romic. Analytical evaluation of the clinical chemistry analyzer Olympus AU2700 plus. Biocehmia Medica, 2010; 20(3): 334-340.

[3] Muser, J, J. Bienvenu, N. Blanckaert, I. Brandslund, J. Delattre, G. Soffiati, R. Swaminathan, S. Maggini, H. Mastall. Inter-Laboratory evaluation of the Cobas Integra 400 analytical system. Clin Chem. Lab. Med, 2001; 39(6): 539559.

[4] Miler, M, A. M. Simundic, M. Stefanovic, D. FerenecRuzic, M. Kvaternik, E. Topic and N. Vrkic, A model for results comparison on two different biochemistry analyzers in laboratory accredited according to the ISO 15189. BiochemiaMedica,2009; 19: 287-293.

[5] ISO 15189: 2012. Medical laboratories. Requirements for quality and competence. International organization for standardization, Geneva, 2012.

[6] ES ISO 15189:2013. Medical laboratories. Requirements for quality and competence. Ethiopian Standard Agency, Addis Ababa. 2007.

[7] CLSI. User Verification of Performance for Precision and Trueness; Approved Guideline-Second Edition. CLSI document EP15-A2. Clinical and Laboratory Standards Institute, Pennsylvania, 2005.

[8] The Westgard Web Lessons. Avaliable at: http://www.westgard.com/lesson.htm. Accessed January 5, 2016.

[9] Ricos C, Perich C, Minchinela J, Alvarez V, Simon M, Biosca $\mathrm{C}$, et al. Application of biological variation - a review. Biochem Med 2009; 19:250-9.

[10] Ricos C, Alvarez V, Cava F, Garcia-Lario JV, Hernandez A, Jimenez CV, et al. Current databases on biologic variation: pros, cons and progress. Scand J Clin Lab Invest 1999; 59: 491-500. This database was most recently updated in 2014. 